\title{
Actitud de Marcha según Nivel Socioeconómico en Adultos Mayores Autovalentes
}

\author{
Paul Medina González ${ }^{1}$ \\ Universidad Católica del Maule
}

\begin{abstract}
RESUMEN - La marcha es fundamental para la funcionalidad de adultos mayores (AM), además su expresión actitudinal se relaciona con diferentes estados emocionales, los cuales son condicionados por el nivel socioeconómico (NSE). El propósito de esta investigación fue evaluar el comportamiento de atributos actitudinales de marcha según el NSE en AM autovalentes. Participaron este estudio 71 AM autovalentes de la comunidad de Talca-Chile, categorizados según el NSE a los cuales se les aplicó el instrumento denominado análisis de forma y esfuerzo de la marcha previo caracterización de confiabilidad. Los resultados indican que el estilo de marcha no es diferente según el NSE en AM autovalentes; sin embargo, los perfiles cualificados presentan variaciones en los anclajes las que son dependientes del género.
\end{abstract}

Palabras-clave: marcha, postura, comunicación no-verbal, factores socioeconómicos, envejecimiento

\section{Gait Attitude according to Socioeconomic Status in Self-reliant Elderly}

\begin{abstract}
The gait is critical to the elderly functionality; in addition, attitudinal expression is related to different emotional states, which are conditioned by socioeconomic status (SES). The purpose of this investigation was to evaluate gait behavior attitudinal attributes according to SES in self-reliant community-dwelling elderly. This study involved 71 self-reliant elderly of Talca-Chile, categorized according to the SES, to whom we applied the shape and effort gait analysis previous reliability characterization. The results indicate that the gait style is not different depending on the SES in self-reliant elderly; however, qualified profiles show variations in anchors which are gender dependent.
\end{abstract}

Keywords: gait, posture, nonverbal communication, socioeconomic factors, aging

Siendo evidente el proceso de transición demográfica hacia estructuras poblacionales envejecidas (Centro Latinoamericano y Caribeño de Demografía, 2006), es que el desafío de instaurar planes para promover la funcionalidad en adultos mayores (AM) resulta fundamental como indicador de desarrollo humano (Huenchuan, 2011). Al respecto, se ha descrito que uno de los elementos centrales para la correcta expresión de funcionalidad y salud, es el estado emocional asociado a las redes sociales del individuo (World Health Organization, 2001), ya que sería el factor regulador, entre otros, de la intencionalidad de movimiento (Medina, Rebolledo, \& Escobar, 2011).

La manifestación motora de las emociones es un área que ha interesado a investigadores que se desempeñan en ambientes de diferente nivel de complejidad biológica y social. Darwin (1872) propone en su obra: "La expresión de las emociones en el hombre y los animales", que esta depende principalmente de características reflejas e instintivas, las cuales se heredan y manifiestan una clara evolución filogenética. Sin embargo, también se describen los hábitos, los cuales son producto de asociaciones entre reflejos, como foco de expresión emocional que pueden modificarse para generar la adaptabilidad del sujeto en su entorno ecológico. La expresión emocional tiene un rol importante en la regulación de interacciones sociales humanas (Maturana, 1989), en este sentido, las emociones son respuestas de múltiples componentes iniciados por cambios

1 Endereço para correspondência: Departamento de Kinesiología, Facultad de Ciencias de la Salud, Universidad Católica del Maule. Avenida San Miguel 3605, Talca, Chile. CEP: 3480112. E-mail: pmedina@ucm.cl en determinadas circunstancias, los cuales desencadenan diferentes tipos de repuestas, incluyendo el movimiento humano (Gross, Crane, \& Fredrickson, 2010; Roether, Omlor, Christensen, \& Glese, 2009). Existe evidencia que vincula al movimiento corporal con la expresión emocional, en este sentido, estudios de neuro-imagen demuestran una relación entre la observación de expresiones corporales y activación de regiones cerebrales que procesan estímulos emocionales y respuesta motora (Prochnow, Steinhäuser, Brunheim, \& Seitz, 2014). En este escenario, se ha planteado en la literatura que el estado emocional estaría vinculado con el estilo de marcha. Al respecto, las sensaciones de enojo, miedo, felicidad y tristeza presentan comportamientos cinemáticos específicos en ubicaciones anatómicas tales como cabeza, columna, hombros, codos, caderas y rodillas (Roether et al., 2009). Complementariamente, una reciente investigación ha demostrado mediante el análisis de espectroscopia funcional por infrarrojo cercano, que existen incrementos en las concentraciones de hemoglobina oxigenada en las regiones corticales occípito-temporal derecha y témporo-parietal izquierda de evaluadores externos al observar avatares anónimos que expresan patrones de marcha temerosos, enojados y tristes (Schneider et al., 2014).

Investigaciones realizadas en AM han documentado que el compromiso emocional, dado principalmente por depresión, tiene mayor predisposición en las últimas etapas del ciclo vital (Djernes, 2006). Al respecto, destaca el modelo bio-psico-social de vulnerabilidad de compromiso y resistencia psicológica en etapas avanzadas del ciclo vital, el cual expone que el estatus funcional psicológico depende de cómo se asimilan durante la vida las cargas dadas por factores 
biológicos, socio-estructurales y las adversidades psicosociales, siendo la capacidad funcional física un elemento importante de resistencia (National Research Council Panel on Race, Ethnicity, and Health in Later Life, 2004), en tal contexto, se suman factores sociales y económicos a la expresión de labilidad emocional, reportándose que su prevalencia es diferente según factores que regulan el comportamiento humano tales como la educación y el acceso a bienes (Ministerio de Salud de Chile, 2010; Rebato, Susanne, \& Chiarelli, 2005), los cuales convergen actualmente en lo que se denomina nivel socioeconómico (NSE). En este escenario, se ha descrito específicamente que la tasa de incidencia para la limitación de movilidad se incrementa dos veces en AM que no poseen bienes y tienen menos de 12 años de escolaridad (Koster et al., 2005). Asimismo la traducción de este fenómeno decanta en una pobre percepción de la calidad de vida en comunidades con vulnerabilidad social y económica (Subramanian, Delgado, Jadue, Vega, \& Kawachi, 2003).

A pesar de la importancia de cuantificar y cualificar la expresión emocional, definida por Maturana (1989) como disposiciones corporales que especifican el dominio de acciones de un ser vivo, se dispone de escasa información en la literatura que confirme modelos confiables y válidos de medición. No obstante, Laban (1974) en su trabajo basado preferentemente en el análisis de bailarinas, propone el modelo de análisis de forma y esfuerzo, el cual contempla componentes y factores para una variedad de contextos de expresión motriz. Siguiendo esta línea Gross et al., (2010) utilizaron este modelo para caracterizar la marcha humana según el comportamiento de los factores de forma denominados cabeza y tronco, además de los de esfuerzo, los cuales describen la utilización del tiempo, flujo, energía y espacio. Posteriormente, este mismo equipo de investigación ha desarrollado estudios exploratorios para describir este comportamiento según diferentes estados emocionales (Crane \& Gross, 2013). Si bien se han reportado resultados de investigaciones metodológicas acerca del modelo de
Análisis de Forma y Esfuerzo de la Marcha (AFEM), el cual se ha sometido a la comprobación de confiabilidad para evaluadores inexpertos (Gross et al., 2010) y validez mediante comparación con perfiles cinemáticos (Gross, Crane, \& Fredrickson, 2012), aun se debe profundizar esta temática dado que se ha implementado solo en grupos de estudiantes universitarios, el cual al ser altamente seleccionado, debilita la extrapolación de los resultados hacia grupos poblacionales vulnerables tales como son los AM.

Entregados estos antecedentes, los propósitos de la presente investigación son en primer lugar determinar la confiabilidad inter-evaluador de la aplicación del AFEM por parte de evaluadores universitarios con instrucción básica, para posteriormente evaluar la expresión emocional a través del comportamiento de la forma y esfuerzo de marcha confortable según el NSE y género en AM chilenos autovalentes de la comunidad.

\section{Método}

\section{Participantes}

Participaron en esta investigación, previa firma de un consentimiento informado, 71 AM pertenecientes a clubes sociales de la comunidad de Talca-Chile. Los criterios de inclusión fueron controlados mediante la aplicación del Examen de Medicina Preventiva del Adulto Mayor (Ministerio de Salud Chile, 2014), verificándose lo siguiente: edad entre 60-75 años, normopeso o sobrepeso según estratificación específica del AM (Estadímetro DETECTO, modelo 2392), autovalentes según la Evaluación Funcional del Adulto Mayor-Chile, parte A (EFAM-Chile), nivel cognitivo normal (Test Minimental abreviado $\geq 13$ puntos) y sin depresión establecida (Escala de Yessavage $<$ cinco puntos). Por su parte, fueron excluidos los sujetos con enfermedades crónicas descompensadas, riesgo de caídas

Tabla 1. Características demográficas y antropométricas de los participantes $(N T=71)$

\begin{tabular}{|c|c|c|c|c|c|c|c|c|c|}
\hline NSE & Género & n & $\begin{array}{l}\text { Edad } \\
\text { (años) }\end{array}$ & $\begin{array}{l}\text { Peso } \\
\text { (kilos) }\end{array}$ & $\begin{array}{c}\text { Talla } \\
\text { (metros) }\end{array}$ & $\begin{array}{c}\text { IMC } \\
\text { (Kilos/m2) }\end{array}$ & $\begin{array}{l}\text { EFAM A } \\
\text { (puntaje) }\end{array}$ & $\begin{array}{l}\text { MMSE } \\
\text { (puntaje) }\end{array}$ & $\begin{array}{c}\text { EDG } \\
\text { (puntaje) }\end{array}$ \\
\hline \multirow{3}{*}{ MB } & $\mathrm{F}$ & 30 & $69 \pm 4$ & $71,8 \pm 9,8$ & $\begin{array}{c}1,52 \pm \\
0,06\end{array}$ & $31,2 \pm 4,3$ & $49 \pm 3$ & $18 \pm 1$ & $4 \pm 3$ \\
\hline & M & 19 & $68 \pm 6$ & $82,4 \pm 12,7$ & $\begin{array}{c}1,64 \pm \\
0,05\end{array}$ & $30,5 \pm 3,1$ & $51 \pm 2$ & $18 \pm 1$ & $4 \pm 2$ \\
\hline & Total & 39 & $69 \pm 5$ & $74,1 \pm 11,3$ & $\begin{array}{c}1,54 \pm \\
0,07\end{array}$ & $31,0 \pm 4,0$ & $49 \pm 3$ & $18 \pm 1$ & $4 \pm 3$ \\
\hline \multirow{3}{*}{ MA } & $\mathrm{F}$ & 26 & $70 \pm 6$ & $70,7 \pm 13,0$ & $\begin{array}{c}1,53 \pm \\
0,06\end{array}$ & $30,2 \pm 4,7$ & $52 \pm 3$ & $18 \pm 1$ & $4 \pm 3$ \\
\hline & M & 6 & $74 \pm 7$ & $81,4 \pm 10,6$ & $\begin{array}{c}1,69 \pm \\
0,07\end{array}$ & $28,5 \pm 4,2$ & $52 \pm 2$ & $18 \pm 1$ & $3 \pm 2$ \\
\hline & Total & 32 & $71 \pm 6$ & $72,9 \pm 13,2$ & $\begin{array}{c}1,56 \pm \\
0,09\end{array}$ & $29,8 \pm 4,6$ & $52 \pm 3$ & $18 \pm 1$ & $3 \pm 3$ \\
\hline
\end{tabular}

Nota. Los valores se expresan en promedios \pm desviación estándar para cada variable. NT = Número Total de participantes; NSE $=$ Nivel Socioeconómico; $\mathrm{MB}=$ Medio Bajo; $\mathrm{MA}=$ Medio Alto; $\mathrm{F}=$ Femenino; $\mathrm{M}=$ Masculino y $\mathrm{n}=$ número de participantes por grupo. IMC = Índice de Masa Corporal; EFAM A = Evaluación Funcional del Adulto Mayor parte A; MMSE = Examen cognitivo Minimental abreviado; EDG = Escala de Depresión Geriátrica 
establecido, secuelas de enfermedades neurológicas o cardiovasculares y dolor moderado de miembros inferiores.

El NSE se determinó mediante la aplicación de la encuesta Adimark (2000), en este sentido se operacionalizó el NSE medio-bajo (MB) para los grupos C3 y D, mientras que el NSE medio-alto (MA) contempló la categorización Abc1 y C2. Las características demográficas, antropométricas y funcionales se presentan en la Tabla 1.

\section{Mediciones}

Posterior a la medición del estado funcional de los AM, se les solicitó que ejecutaran una marcha confortable durante tres minutos en un circuito elíptico de 40 metros (Figura 1A), este tipo de marcha se ha descrito como la más cercana a contextos de funcionalidad cotidianos para el desarrollo de actividades de la vida diaria (Varela, Ortiz, \& Chávez, 2010), al respecto es considerada como una marcha en condiciones naturales, sin una exigencia o incentivo superior al funcional. Se ubicó estratégicamente una cámara (Sony Handycam, modelo HDR-XR550) en una zona denominada "de registro", a una distancia de cuatro metros para la captura de un video de cada zancada ejecutada por el AM (Figura 1B). Una vez terminada la ejecución de la prueba, se guardaron los registros en un computador portátil (TOSHIBA, modelo NB505-SP0115LL) para el desarrollo de una sesión de análisis por parte de evaluadores voluntarios y externos a la investigación, los cuales fueron previamente instruidos en la aplicación del instrumento de calificación. La Figura $1 \mathrm{C}$ muestra un fotograma representativo del video analizado.

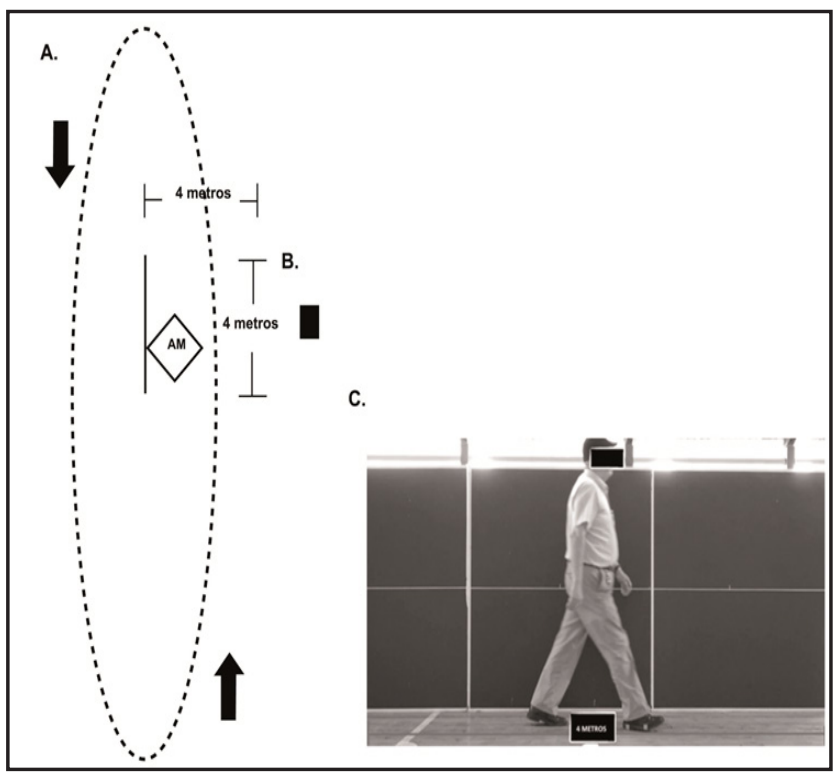

Figura 1. Captura y registro de la información

A. Circuito de 40 metros para el desarrollo de marcha confortable del adulto mayor (AM). B. Ubicación de cámara fija y delimitación de zona de registro para la captura del video. C. Fotograma que representa la vista de los evaluadores en un plano sagital espacial con las longitudes del panel de referencia. Las flechas indican la dirección del movimiento.

\section{Instrumento y evaluadores}

Se utilizó un instrumento tipo escala de Likert denominado AFEM (Figura 2) según el protocolo declarado en su propuesta original (Gross et al., 2010). Al respecto, la operacionalización de los componentes de forma se enmarca en la cinemática descriptiva de la postura de tronco en el plano sagital corporal, además de la amplitud del movimiento desarrollado por los brazos. Por su parte, el componente esfuerzo contempla la utilización de los anclajes o extremos descriptivos para los factores: espacio errante-enfocado, el comportamiento de energía mediante el movimiento liviano-pesado, el tiempo a través de la expresión de marcha sostenida-súbita y finalmente el flujo mediante la dualidad marcha relajada-tensa.

Para el desarrollo del AFEM, se prepararon videos de cada pasaje del AM por la zona de registro, el cual fue analizado por todos los evaluadores en una sala pertinentemente preparada. Cabe destacar que cada evaluador observó una vez el video para el análisis de cada factor declarado en el instrumento.

Antes de efectuar el análisis del comportamiento de marcha, se realizó un estudio para determinar el nivel consistencia y acuerdo existente entre evaluadores universitarios con calificación suficiente en la aplicación del instrumento. Esta comprendió en primera instancia el control teórico de la escala y sus principios, luego se realizó una aplicación piloto asistida, para finalmente aplicar el modelo de manera autónoma. Participaron en el estudio todos los estudiantes que obtuvieron en su rúbrica de calificación la habilitación básica. En este escenario, para el estudio de confiabilidad

\begin{tabular}{|c|c|c|c|c|c|c|}
\hline COMPONENTES & FACTORES & \multicolumn{5}{|c|}{ CRITERIOS DE CATEGORIZACIÓN } \\
\hline \multirow{4}{*}{ I.- FORMA } & la.-Tronco & $\begin{array}{l}\text { Postura con } \\
\text { Inclinación } \\
\text { anterior }\end{array}$ & & & & $\begin{array}{c}\text { Postura de } \\
\text { tronco con } \\
\text { inclinación } \\
\text { posterior }\end{array}$ \\
\hline & Puntaje & 1 & 2 & 3 & 4 & 5 \\
\hline & lb.- Brazos & \multicolumn{4}{|l|}{$\begin{array}{l}\text { Amplitud de } \\
\text { movimiento } \\
\text { fruncido y } \\
\text { encogido }\end{array}$} & $\begin{array}{l}\text { Amplitud de } \\
\text { movimiento } \\
\text { extendido y } \\
\text { crecido }\end{array}$ \\
\hline & Puntaje & 1 & 2 & 3 & 4 & 5 \\
\hline \multirow{8}{*}{ II.- ESFUERZO } & Ila.- Espacio & \multicolumn{4}{|l|}{$\begin{array}{c}\text { Movimiento } \\
\text { errante y difuso }\end{array}$} & $\begin{array}{l}\text { Movimiento } \\
\text { enfocadoy } \\
\text { canalizado }\end{array}$ \\
\hline & Puntaje & 1 & 2 & 3 & 4 & 5 \\
\hline & Ilb.- Energia & \multicolumn{4}{|l|}{$\begin{array}{l}\text { Movimiento } \\
\text { livianoy } \\
\text { flotanté }\end{array}$} & $\begin{array}{l}\text { Movimiento } \\
\text { pesado }\end{array}$ \\
\hline & Puntaje & 1 & 2 & 3 & 4 & 5 \\
\hline & IIc-Tiempo & \multicolumn{4}{|l|}{$\begin{array}{c}\text { Marcha } \\
\text { sostenida y } \\
\text { pausada }\end{array}$} & $\begin{array}{c}\text { Marcha súbita y } \\
\text { brusca }\end{array}$ \\
\hline & Puntaje & 1 & 2 & 3 & 4 & 5 \\
\hline & Ild.- Flujo & \multicolumn{4}{|l|}{$\begin{array}{l}\text { Marcha libre y } \\
\text { telajada }\end{array}$} & $\begin{array}{c}\text { Marcha tensa y } \\
\text { aprisionada }\end{array}$ \\
\hline & Puntaje & 1 & 2 & 3 & 4 & 5 \\
\hline
\end{tabular}

Figura 2. Instrumento utilizado para la calificación de la forma y esfuerzo de la marcha

El componente denominado forma se divide en los factores tronco y brazos, mientras que el componente esfuerzo considera espacio, energía, tiempo y flujo. El análisis se desarrolla mediante una metodología de categorización tipo escala de Likert. 
inter-evaluador participaron en total 20 evaluadores, de los cuales 10 analizaron en una primera etapa los registros de la totalidad de AM participantes $(n=71)$, siendo este mismo análisis desarrollado por los demás $(n=10)$, obteniéndose el análisis de dos evaluadores por cada participante.

\section{Análisis estadístico}

El manejo descriptivo de las variables representativas del estado antropométrico y funcional de los AM, fue mediante promedio y \pm una desviación estándar. En el caso de los factores del AFEM, se utilizó moda y frecuencia relativa (porcentaje).

La prueba de confiabilidad comprendió la evaluación de la reproducibilidad del instrumento mediante la prueba Tau-b de Kendall y la determinación del grado acuerdo a través del índice Kappa de Cohen. Para comparar los factores de forma y esfuerzo de marcha según el NSE se recurrió a la prueba U Mann Whitney. El nivel de significancia estadística se estableció en un $\mathrm{p} \leq 0,05$. Para el análisis de datos se utilizó en programa PASW Statistics versión 18.0.

\section{Resultados}

Las características demográficas, antropométricas y funcionales de los participantes se resumen en la Tabla 1. En la mayoría de los AM, el rango etario comprende el decenio 65-75 años, siendo predominante el estado nutricional normopeso y sobrepeso para ambos NSE operacionalizados. Además, los sujetos de evaluación pertenecen a la estratificación autovalentes, sin riesgo de depresión y un estado cognitivo normal.

Los resultados de confiabilidad inter-evaluador se exponen en la Tabla 2. En este sentido, la reproducibilidad resulta significativa en todos los factores analizados, obteniéndose valores desde 0,25 en el caso de energía $(p<0,05)$ hasta al menos 0,59 en los demás factores $(\mathrm{p}<0,001)$. Por su parte, el grado de acuerdo muestra índices mayores a 0,45 en todos los factores a excepción de energía $(\mathrm{p}<0,001)$.

Tabla 2. Acuerdo y consistencia inter-evaluador de la actitud de marcha confortable $(n=71, k=2)$

\begin{tabular}{lcccc}
\hline $\begin{array}{c}\text { Factores } \\
\text { forma y } \\
\text { esfuerzo }\end{array}$ & Reproducibilidad & $\mathbf{p}$ & Acuerdo & $\mathbf{p}$ \\
\hline Tronco & 0,73 & $* * *$ & 0,59 & $* * *$ \\
Brazos & 0,83 & $* * *$ & 0,67 & $* * *$ \\
Energía & 0,25 & $*$ & 0,07 & NS \\
Espacio & 0,59 & $* * *$ & 0,51 & $* * *$ \\
Tiempo & 0,70 & $* * *$ & 0,55 & $* * *$ \\
Flujo & 0,56 & $* * *$ & 0,47 & $* * *$ \\
\hline
\end{tabular}

Nota: $\mathrm{n}=$ número de sujetos participantes $\mathrm{y} \mathrm{k}=$ número de evaluadores. La reproducibilidad de medición interevaluador fue desarrollada mediante la aplicación de la prueba Tau-b de Kendall. El grado de acuerdo entre evaluadores se ejecutó mediante índice Kappa de Cohen. $\mathrm{NS}=$ No existen diferencias significativas $* p<, 05 ; * * * p<, 001$
El comportamiento de la marcha confortable mediante el análisis de forma y esfuerzo según NSE y género es descrito en la Tabla 3, no expresando diferencias significativas. No obstante, el perfil actitudinal expuesto en la Figura 3 da cuenta de una mayor utilización del "espacio" en el NSE MA para tanto el grupo general de análisis (Figura 3A) como mujeres (Figura 3B), traduciéndose en una marcha canalizada-enfocada. Además, específicamente en el género femenino se aprecia una utilización del "flujo" con anclaje derecho dando cuenta de una marcha tensa y aprisionada (Figura 3B).

En el género masculino, el grupo de AM pertenecientes al NSE MB reporta un anclaje izquierdo para el componente de forma denominado "tronco", traduciendo una inclinación anterior. Además, anclaje derecho para los componentes de esfuerzo denominados "espacio", "energía” y "tiempo", traduciendo una marcha de características enfocada-canalizada, pesada y súbita. Finalmente, en el caso del NSE MA la marcha es de características neutra (Figura 3C).

\section{Discusión}

Este es el primer estudio que propone evaluar la expresión emocional mediante el comportamiento de los componentes de forma y esfuerzo de marcha confortable en AM autovalentes de la comunidad. Dentro de este escenario metodológico, es que el propósito inicial de esta investigación fue describir el desempeño de la confiabilidad inter-evaluador al aplicar el AFEM en AM autovalentes, al respecto, los resultados expresan niveles de acuerdo y consistencia aceptables, así como similares a los reportados por la línea de investigación original (Gross et al., 2010), lo que garantiza comenzar el análisis con una base suficiente (Tabla 2). Cabe destacar, que Tabla 3. Factores de forma y esfuerzo de marcha confortable según NSE y género

\begin{tabular}{ccccc}
\hline \multirow{2}{*}{ Género } & $\begin{array}{c}\text { Factores } \\
\text { de } \\
\text { Forma y } \\
\text { Esfuerzo }\end{array}$ & $\begin{array}{c}\text { NSE } \\
\text { Medio } \\
\text { Bajo }\end{array}$ & $\begin{array}{c}\text { NSE } \\
\text { Medio } \\
\text { Alto }\end{array}$ & Valor p \\
\hline \multirow{7}{*}{ Femenino } & Tronco & $2(33,3 \%)$ & $3(57,7 \%)$ & $0,483 \mathrm{NS}$ \\
& Brazos & $2(36,7 \%)$ & $2(38,5 \%)$ & $0,665 \mathrm{NS}$ \\
& Espacio & $3(43,3 \%)$ & $4(46,2 \%)$ & $0,103 \mathrm{NS}$ \\
& Energía & $3(36,7 \%)$ & $3(38,5 \%)$ & $0,601 \mathrm{NS}$ \\
& Tiempo & $3(53,3 \%)$ & $3(34,6 \%)$ & $0,890 \mathrm{NS}$ \\
& Flujo & $4(36,7 \%)$ & $4(26,9 \%)$ & $0,087 \mathrm{NS}$ \\
& Tronco & $2(37,5 \%)$ & $3(57,1 \%)$ & $0,396 \mathrm{NS}$ \\
& Brazos & $3(62,5 \%)$ & $3(57,1 \%)$ & $0,511 \mathrm{NS}$ \\
& Espacio & $4(50,0 \%)$ & $3(57,1 \%)$ & $0,998 \mathrm{NS}$ \\
& Energía & $4(37,5 \%)$ & $3(71,4 \%)$ & $0,203 \mathrm{NS}$ \\
& Tiempo & $4(37,5 \%)$ & $3(57,1 \%)$ & $0,123 \mathrm{NS}$ \\
& Flujo & $3(50,0 \%)$ & $3(57,1 \%)$ & $0,705 \mathrm{NS}$ \\
\hline
\end{tabular}

Nota: Valores de categorización para cada atributo: $1=$ anclaje izquierdo extremo; $2=$ anclaje izquierdo; $3=$ neutro; 4 = anclaje derecho y 5 = anclaje derecho extremo. Los valores de categorización se expresan en moda con su correspondiente frecuencia relativa porcentual de los casos válidos en paréntesis. NS $=$ No existen diferencias significativas. 
la energía resulta ser el factor más discutible. Tal vez esta situación se explica por las características de los descriptores, los cuales mencionan para este factor un espectro que va desde un movimiento liviano y delicado hasta el movimiento pesado (Figura 2), los cuales se podrían confundir dadas las características antropométricas de los participantes analizados (Laus, Almeida, Murarole, \& Braga-Costa, 2013).

El análisis de forma y esfuerzo proviene de la propuesta diseñada por Laban (1974), en este contexto, la aplicación de estos principios asociados al comportamiento de marcha confortable presenta una línea de trabajo investigativo creciente. En el presente estudio, se describió mediante la apreciación de evaluadores externos, la forma (cualidad cinemática) y el esfuerzo (cualidad témporo-espacial) de la marcha confortable en AM autovalentes. Los resultados obtenidos reflejan una tendencia incipiente hacia estilos de marcha de mayor o menor anclaje según NSE y género (Figura 3), no obstante lo anterior, esta aparente divergencia perceptual no resulta ser estadísticamente significativa (Tabla 3). Cabe destacar, que la divergencia más acentuada asociada al estilo de marcha involucra el espacio, el cual es de anclaje derecho en el NSE MA, lo que se traduce en una marcha canalizada-enfocada, siendo en el NSE MB de características neutras (Tabla 3, Figura 3A). En este contexto, los reportes de la literatura tienen como propósito analizar los patrones cinemáticos de marcha según la expresión emocional (Crane, \& Gross, 2013; Barliya, Omlor, Giese, Berthoz, \& Flash, 2013), entregando resultados de estilos de marcha que son particulares según el comportamiento cinemático de esqueleto axial y apendicular. Al respecto, la marcha de tipo canalizada para la utilización del espacio se asocia con expresiones emocionales de enojo a alegría, no siendo específica para cada una de ellas.

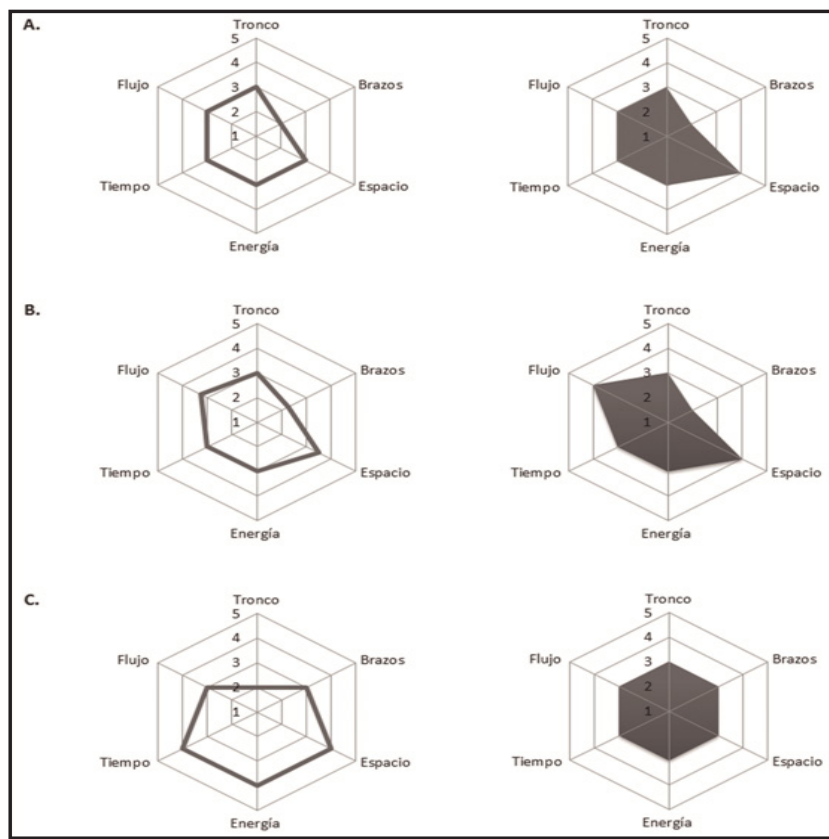

Figura 3. Actitud de marcha confortable según NSE y género Gráficos radiales que representan la moda de los factores de forma y esfuerzo de la marcha según NSE para: A. Participantes en general. B. Género femenino y C. Género masculino. Los sectores sin relleno representan el NSE MB, mientras que los oscuros el NSE MA.
Ahora bien, si consideramos el género como segundo factor de análisis, Barliya et al. (2013), determinaron las características de los estilos de movimiento durante marcha confortable en relación a diferentes emociones, en este contexto, si vinculamos esta evidencia con los resultados obtenidos según el NSE, encontramos que los individuos del género femenino pertenecientes al NSE MA expresan una marcha confortable con utilización de flujo y espacio hacia el anclaje derecho, esto es, canalizada-enfocada con un flujo aprisionado (Tabla 3, Figura 3B), lo que se traduce en una caracterización emocional de "molestia" (Crane \& Gross, 2013). Esto se puede explicar por lo prolongado de la medición, la que en algunos casos era cercana a una hora de trabajo en condiciones de temperatura no siempre controlada, en consecuencia, este factor deberá ser considerado en futuras investigaciones. Por su parte, en hombres ocurre que las mayores diferencias se dan en el NSE MB, el cual manifiesta anclaje derecho para el tiempo, energía y espacio, mientras que se aprecia anclaje izquierdo en la forma del tronco (Tabla 3, Figura 3C). Este comportamiento traduce una marcha de características súbita, pesada y canalizada con una postura axial hacia anterior, demostrando un estado emocional preferente de "entretención" (Crane \& Gross, 2013). Cabe destacar que aquella disimilitud se podría explicar por la mayor variabilidad lineal de la velocidad promedio de marcha expresada en este grupo socioeconómico (Tabla 4).

Los resultados obtenidos con esta experiencia proponen que los AM pertenecientes al NSE MA presentan expresiones emocionales negativas a neutras, mientras que en la posición socioeconómica baja es de predominio preferentemente positivo. Al respecto, se dispone de información discrepante en la literatura, la cual mediante un modelo conceptual que explica la asociación dinámica entre el NSE bajo y el entorno, plantea que tanto las emociones como las capacidades cognitivas negativas tendrían mayor expresión en ambientes pobres y de mayor vulnerabilidad, dado la baja disposición de recursos y la disminuida posición en la jerarquía social (Gallo \& Matthews, 2003). Este comportamiento desigual se podría explicar en el contexto de que las mediciones fueron efectuadas en condiciones nulas de asistencialismo, lo que garantiza un grato ambiente de trabajo, buen trato y la implementación de los tiempos necesarios para educar y aclarar dudas a los AM durante las evaluaciones, al respecto estos estándares de práctica al ser cotidianos para el sistema privado no significarían una ventaja comparativa para los participantes del NSE MA, por otro lado, los AM del NSE MB apreciarían esta experiencia, expresando una actitud de marcha más positiva ante la actividad requerida. Por otro lado, la homogeneidad de la muestra estudiada en relación a la reserva funcional y psicológica (Tabla 1) podría explicar el comportamiento estadístico similar en los resultados, en este sentido, esta aseveración se fundamenta en que además de los aspectos sociales, también son factores desencadenantes de labilidad o resiliencia psicológica los elementos biológicos y la experiencia de adversidades psico-sociales (National Research Council Panel on Race, Ethnicity, and Health in Later Life, 2004). Es importante mencionar que estos resultados son preliminares y por tanto los datos que emergen deben ser tomados con cautela, proyectándose la medición 
Tabla 4. Velocidad promedio y su variabilidad lineal según NSE y género

\begin{tabular}{cccccc}
\hline $\begin{array}{c}\text { Velocidad } \\
\text { Promedio }\end{array}$ & Género & NSE MB & \%CV NSE MB & NSE MA & \%CV NSE MA \\
\hline Marcha & F & $1,10 \pm 0,19$ & 17,3 & $1,16 \pm 0,18$ & 15,5 \\
Confortable & M & $1,22 \pm 0,19$ & 15,6 & $1,16 \pm 0,07$ & 6,0 \\
$(\mathrm{~m} / \mathrm{s})$ & Total & $1,13 \pm 0,19$ & 16,8 & $1,16 \pm 0,16$ & 13,8 \\
\hline
\end{tabular}

Nota: Los valores se expresan en promedios \pm desviación estándar para cada variable. NSE $\mathrm{MB}=$ nivel socioeconómico medio-bajo, NSE MA = nivel socioeconómico medioalto. $\% \mathrm{CV}=$ porcentaje del coeficiente de variación como indicador de variabilidad lineal.

en otros grupos de sujetos considerando la manifestación de variabilidad etaria, antropométrica, funcional y psicológica.

Analizando la percepción del movimiento por parte de evaluadores externos, esta es una variable que en investigaciones anteriores no ha sido considerada para el análisis, al respecto se ha documentado que esta apreciación depende de mecanismos neuronales específicos para el reconocimiento del movimiento biológico, mediante neuronas localizadas tanto en el surco temporal superior como la corteza pre-motora, las cuales tendrían características de funcionalidad multimodal, otorgando la capacidad de responder a los aspectos visuales, espaciales y sonoros de este gesto motor (Giese \& Poggio, 2003). Cabe destacar, que si bien los evaluadores al ser estudiantes universitarios, poseían características físicas y demográficas similares, no se analizaron los aspectos concernientes a su estado sensorial, por lo que debería ser un elemento importante al momento de determinar las características de inclusión para todo sujeto que evalúe y cualifique el movimiento, siendo este razonamiento válido para diferentes experiencias tales como la calificación en la gimnasia, saltos ornamentales, baile o danza.

Si consideramos el impacto que podría tener el ambiente sobre la expresión corporal, existen aspectos relacionados al aprendizaje y la planificación motora mediante la observación, los cuales dependen de grupos de neuronas localizadas en la zona F5 de la corteza pre-motora, denominadas complejo de neuronas espejo (Oberman, Pineda, \& Ramachandran, 2007), las cuales podrían explicar, en parte, los diferentes estilos de marcha según los contextos socio-culturales.

El AFEM ha demostrado ser una herramienta confiable de calificación para la actitud de marcha por parte de evaluadores con instrucción básica, no obstante, el factor energía debe evitar el sesgo que produce la observación del estado nutricional de los sujetos. Además, esta instancia de valoración dependería de los marcos teóricos que disponen los sujetos que califican, por lo que conceptualizaciones del rendimiento y variabilidad cinemática relacionada al proceso de envejecimiento (Hamacher, Singh, Van Dieën, Helle, \& Taylor, 2011), además del género como factor (Biomotion Lab, 2013) podrían condicionar la cualificación.

Dentro de las limitaciones del estudio se encuentra el bajo tamaño muestral, además de que la selección de sujetos fue realizada por conveniencia. Por otro lado, la caracterización del componente emocional basal de los sujetos consideró solo aspectos vinculados con la depresión, dejando de lado factores significativos del estado emocional tales como la presencia de ansiedad o estrés. En este contexto, se proyecta incorporar esta metodología de análisis en futuras investi- gaciones que contemplen junto con un mayor número de sujetos, la expresión de variabilidad en aspectos funcionales, psicológicos y sociales de los AM participantes.

En consecuencia a los resultados obtenidos, la cuantificación del comportamiento de los factores de forma $\mathrm{y}$ esfuerzo de marcha confortable no es diferente según el NSE en AM autovalentes de la comunidad. Por otro lado, los perfiles cualificados presentan variaciones traducidas en diferentes anclajes según el NSE, las cuales son dependientes del género, de esta manera, los AM pertenecientes al NSE MA presentan una expresión emocional negativa en mujeres a neutra en hombres, mientras que los del NSE MB presentan anclajes concordantes con percepciones positivas en ambos géneros.

\section{Referencias}

Adimark. (2000). Mapa socioeconómico de Chile: nivel socioeconómico del país basado en los datos del Censo. Retrieved from http://www.adimark.cl/medios/estudios/ mapa_socioeconomico_de_chile.pdf

Barliya, A., Omlor, L., Giese, M.A., Berthoz, A., \& Flash, T. (2013). Expression of emotion in the kinematics of locomotion. Experimental Brain Research, 225(2), 159-176. doi: 10.1007/ s00221-012-3357-4.

Biomotion Lab. (2013). Analysis and Synthesis of Biological Motion Patterns. Retrieved from http://www.biomotionlab. ca/walking.php

Centro Latinoamericano y Caribeño de Demografía - División de Población de la CEPAL. (2006). Manual sobre indicadores de la calidad de vida en la vejez: Demografía del envejecimiento. Retrieved from http://www.eclac.cl/cgibin/getProd.asp?xml=/ publicaciones/xml/0/28240/P28240.xml\&xsl=/celade/tpl/p9f. $\mathrm{xsl} \&$ base $=/$ celade/tpl/top-bottom.xsl

Crane, E., \& Gross, M. (2013). Effort-shape characteristics of emotion-related body movement. Journal of Nonverbal Behavior, 37(2), 91-105. doi: 10.1007/s10919-013-0144-2

Darwin, C. (1872). The expression of emotions in man and animals. Retrieved from https://archive.org/details/ expressionofemot1872darw

Djernes, J. K. (2006). Prevalence and predictors of depression in populations of elderly: a review. Acta Psychiatrica Scandinavica, 113(5), 372-387. doi: 10.1111/j.16000447.2006.00770.x 
Gallo, L., \& Matthews, K. (2003). Understanding the association between socioeconomic status and physical health: do negative emotions play a role? Psychological Bulletin, 129(1), 10-51. doi: 10.1037/0033-2909.129.1.10

Giese, M. A., \& Poggio, T. (2003). Neural mechanisms for the recognition of biological movements. Nature Reviews Neuroscience, 4(3), 179-192. doi: 10.1038/nrn1057

Gross, M., Crane, E., \& Fredrickson, B. (2010). Methodology for assessing bodily expression of emotion. Journal of Nonverbal Behavior, 34(4), 223-248. doi: 10.1007/s10919-010-0094-x

Gross, M., Crane, E., \& Fredrickson, B. (2012). Effort-Shape and kinematic assessment of bodily expression of emotion during gait. Human Movement Science, 31(1), 202-221. doi: 10.1016/j. humov.2011.05.001.

Hamacher, D., Singh, N.B., Van Dieën, J.H., Heller, M.O., \& Taylor, W.R. (2011). Kinematic measures for assessing gait stability in elderly individuals: a systematic review. Journal of the Royal Society Interface. 8(65), 1682-1698. doi: 10.1098/ rsif.2011.0416

Huenchuan, S. (2011). La protección de salud en el marco de la dinámica demográfica de los derechos. Serie población y desarrollo - Comisión Económica para América Latina y el Caribe. Retrieved from http://www.eclac.org/cgi-bin/ getProd.asp?xml=/publicaciones/xml/3/43173/P43173. $\mathrm{xml} \& \mathrm{xsl}=/$ publicaciones/ficha-i.xsl\&base=/publicaciones/ top publicaciones-i.xsl

Koster, A., Penninx, B., Bosma, H., Kempen, G., Harris, T., Newman, A. B., ...Kritchevsky, S. B. (2005). Is there a biomedical explanation for socioeconomic differences in incident mobility limitation? Journal of Gerontology Medical Sciences. 60A(8), 1022-1027. doi: 10.1093/gerona/60.8.1022

Laban, R. (1974). The language of movement: A guidebook to choreutics. Boston: Plays, Inc.

Laus, M. F., Almeida, S. S., Murarole, M. B., \& Braga-Costa, T. M. (2013). Estudo de validação e fidedignidade de escalas de silhuetas brasileiras em adolescentes. Psicologia: Teoria e Pesquisa, 29(4), 403-409. doi: 10.1590/S010237722013000400006.

Maturana, H. (1989). Lenguaje y realidad: El origen de lo humano. Archivos de Biología y Medicina Experimental, 22(2), 77-81.

Medina, P., Rebolledo, I., \& Escobar, M. (2011). Operacionalización del modelo función disfunción: Un acercamiento hacia la pretensión de la autonomía profesional. Kinesiología, 30(3), 46-57.

Ministerio de Salud Chile. Programa de Salud del Adulto Mayor. División de Prevención y Control de Enfermedades. Subsecretaría de Salud Pública. (2014). Manual de aplicación del Examen de Medicina Preventiva del Adulto Mayor (EMPAM). Retrieved from http://www.saludohiggins.cl/ attachments/314_Instructivo\%20del\%20Control $\% 20 \mathrm{de} \% 20$ Salud\%200107.pdf
Ministerio de Salud de Chile. (2010). Encuesta Nacional de Salud 2009-2010. Retrieved from http://web.minsal.cl/portal/url/ item/bcb03d7bc28b64dfe040010165012d23.pdf

National Research Council (2004). Panel on Race, Ethnicity, and Health in Later Life. In N. B. Anderson, R. A. Bulatao, \& B. Cohen (Eds.), Critical Perspectives on Racial and Ethnic Differences in Health in Late Life (13). Washington (DC): National Academies Press.Retrieved from http://www.ncbi. nlm.nih.gov/books/NBK25520/

Oberman, L. M., Pineda, J. A., \& Ramachandran, V. S. (2007). The human mirror neuron system: a link between action observation and social skills. Social Cognitive and Affective Neuroscience, 2(1), 62-66. doi: 10.1093/scan/ns1022

Prochnow, D., Steinhäuser, L., Brunheim, S., \& Seitz, R. J. (2014). Differential Emotional State Reasoning in young and older adults: Evidence from behavioral and neuroimaging data. Journal of Neurology and Psychology, 2(1), 1-8. Retrieved from http://www.avensonline.org/wp-content/uploads/2014/08/JNP2332-3469-02-0009.pdf

Rebato, E., Susanne, C., \& Chiarelli, B. (2005). Para comprender la antropología biológica. Evolución y biología humana $\left(1^{\mathrm{a}}\right.$ ed). Navarra: Editorial Verbo Divino.

Roether, C., Omlor, L., Christensen, A., \& Glese, M. (2009). Criticalm features for the perception of emotion from gait. Journal of Vision, 9(6), 15: 1-32. doi: 10.1167/9.6.15.

Schneider, S., Christensen, A., Häußinger, F. B., Fallgatter, A. J., Giese, M. A., \& Ehlis, A. C. (2014). Show me how you walk and I tell you how you feel - a functional nearinfrared spectroscopy study on emotion perception based on human gait. Neuroimage, 85(1), 380-390. doi: 10.1016/j. neuroimage.2013.07.078.

Subramanian, S., Delgado, I., Jadue, L., Vega, J., \& Kawachi, I. (2003). Income inequality and health: multilevel analysis of Chilean communities. Journal of Epidemiology and Community Health, 57(11), 844-848. doi:10.1136/jech.57.11.844

Varela, L., Ortiz, P., \& Chávez, H. (2010). Velocidad de la marcha como indicador de fragilidad en adultos mayores de la comunidad en Lima, Perú. Revista Española de Geriatría y Gerontología, 45(1), 22-25. doi:10.1016/j.regg.2009.07.011

World Health Organization. (2001). The International Classification Functioning, Disability and Health. Retrieved from http:// www.who.int/classifications/icf/en/

Recebido em 25.06.2014

Primeira decisão editorial em 01.11.2014

Versão final em 15.05.2015

Aceito em 16.05.2015 